\title{
Ligand-induced conformational capture of a synthetic tetracycline riboswitch revealed by pulse EPR
}

\author{
DORITH WUNNICKE, ${ }^{1}$ DENISE STROHBACH, ${ }^{2,3}$ JULIA E. WEIGAND, ${ }^{4}$ BETTINA APPEL, ${ }^{5}$ EMILIANO FERESIN, ${ }^{1,6}$ \\ BEATRIX SUESS, ${ }^{4}$ SABINE MÜLLER, ${ }^{5}$ and HEINZ-JÜRGEN STEINHOFF ${ }^{1}$ \\ ${ }^{1}$ Fachbereich Physik, Universität Osnabrück, 49069 Osnabrück, Germany \\ ${ }^{2}$ Fakultät für Chemie und Biochemie, Ruhr-Universität Bochum, 44780 Bochum, Germany \\ ${ }^{3}$ Biomers.net GmbH, Söflinger Str. 100, 89077 Ulm, Germany \\ ${ }^{4}$ Institut für Molekulare Biowissenschaften, Johann Wolfgang Goethe-Universität Frankfurt/Main, 60438 Frankfurt am Main, Germany \\ ${ }^{5}$ Institut für Biochemie, Ernst-Moritz-Arndt-Universität Greifswald, 17487 Greifswald, Germany \\ ${ }^{6}$ Scuola Internazionale Superiore di Studi Avanzati, 34014 Trieste, Italy
}

\begin{abstract}
RNA aptamers are in vitro-selected binding domains that recognize their respective ligand with high affinity and specificity. They are characterized by complex three-dimensional conformations providing preformed binding pockets that undergo conformational changes upon ligand binding. Small molecule-binding aptamers have been exploited as synthetic riboswitches for conditional gene expression in various organisms. In the present study, double electron-electron resonance (DEER) spectroscopy combined with site-directed spin labeling was used to elucidate the conformational transition of a tetracycline aptamer upon ligand binding. Different sites were selected for post-synthetic introduction of either the (1-oxyl-2,2,5,5tetramethylpyrroline-3-methyl) methanethiosulfonate by reaction with a 4-thiouridine modified RNA or of 4-isocyanato-2,6tetramethylpiperidyl-N-oxid spin label by reaction with 2 '-aminouridine modified RNA. The results of the DEER experiments indicate the presence of a thermodynamic equilibrium between two aptamer conformations in the free state and capture of one conformation upon tetracycline binding.
\end{abstract}

Keywords: tetracycline aptamer; synthetic riboswitch; DEER; ESR; site-directed spin labeling; RNA folding

\section{INTRODUCTION}

Riboswitches are regulatory elements, which are primarily located in the $5^{\prime}$-untranslated region (UTR) of prokaryotic mRNAs. They are complex folded single-stranded RNA structures and can bind specific ligands like magnesium ions, cofactors, amino acids, or nucleobases. Ligand binding causes conformational changes that regulate gene expression, either through premature termination of transcription or inhibition of translation initiation (Roth and Breaker 2009). Furthermore, aptamers exhibit properties comparable to antibodies (Gold et al. 1995; Wilson and Szostak 1999). They are potential agents for disease diagnosis and treatment (Famulok et al. 2007) and for biotechnological applications like biosensors (Weigand and Suess 2009). Aptamers are also used as riboswitches for conditional gene

Reprint requests to: Heinz-Jürgen Steinhoff, Fachbereich Physik, Universität Osnabrück, Barbarastr. 7, 49069 Osnabrück, Germany; e-mail: hsteinho@uni-osnabrueck.de; fax: 49-541-969-2656.

Article published online ahead of print. Article and publication date are at http://www.rnajournal.org/cgi/doi/10.1261/rna.2222811. regulation (Suess and Weigand 2008; Win et al. 2009). Such RNA molecules are isolated by SELEX (Systematic Evolution of Ligands by EXponential Enrichment) from a random pool of RNA molecules (Ellington and Szostak 1990; Tuerk and Gold 1990). They specifically bind to previously defined ligands with high affinity and selectivity. This class of nucleic acid molecules adopts complex three-dimensional conformations to provide preformed binding pockets and clefts for the specific recognition and binding of their ligands, such as cofactors, amino acids, drugs, or antibiotics (Famulok 1999; Patel and Suri 2000). Ligand binding is often accompanied by structural rearrangement of the RNA aptamer (Hermann and Patel 2000).

The tetracycline (Tc)-binding aptamer investigated in the present work has been used as an RNA switch for conditional gene expression in yeast, either inhibiting translation initiation (Suess et al. 2003; Kötter et al. 2009) or pre-mRNA splicing (Weigand and Suess 2007). The ligand Tc inhibits prokaryotic translation (Epe et al. 1987; Spahn and Prescott 1996) and is used as a therapeutic agent of low toxicity (Berens and Hillen 2003). The aptamer is biochemically well characterized; it binds its ligand with a dissociation constant 
of $\sim 0.8 \mathrm{nM}$ and has a stoichiometry of one molecule of Tc and one molecule of the prestructured RNA aptamer (Müller et al. 2006). The RNA aptamer comprises three helices (stems $\mathrm{P} 1, \mathrm{P} 2$, and $\mathrm{P} 3$ ), the single-stranded joining regions J1-2 and J2-3 and the loop L3 (Fig. 1; Hanson et al. 2005; Xiao et al. 2008). All three stem regions are not in direct contact with Tc and form the scaffold already in the absence of the ligand. An irregular helix consisting of the regions J1-2 and J2-3 possesses tertiary contacts to nucleotides from L3 in a pseudoknot-like manner. The overall structure resembles that of an inverted " $h$ " with Tc bound at the junction (cf. Fig. 1). However, the nature of the putative Tc-induced conformational transition is still unknown.

Both electron paramagnetic resonance (EPR) spectroscopy in combination with site-directed spin labeling (SDSL) and Förster resonance energy transfer (FRET) spectroscopy allow inter- and intramolecular distance measurements on the nanometer scale. FRET is based on the nonradiative energy transfer between two fluorophors, which permit distance determination in the range of 1-10 nm (Lorenz and Diekmann 2006). Furthermore, single-molecule FRET provides information about the conformational dynamics of single biomolecules over time scales in the range of microseconds to seconds (Kim et al. 2002; Margittai et al. 2003). The precision of this method is impaired by the uncertainty of the orientation factor and by the size of the fluorophors. EPR spectroscopy in combination with SDSL has been shown to be ideally suited to elucidate the conformations and conformational dynamics of biological systems such as proteins, protein complexes, DNA, and RNA under physiological conditions (Hubbell et al. 2000; Cai et al. 2006; Bordignon and Steinhoff 2007). By means of spin labeled folded DNA or RNA a nanometer distance ruler was developed (Schiemann et al. 2003, 2004; Piton et al. 2005), B/A conformational transitions of DNA (Sicoli et al. 2008), and DNA damages (Sicoli et al. 2009) were studied by double electron-electron resonance (DEER) spectroscopy

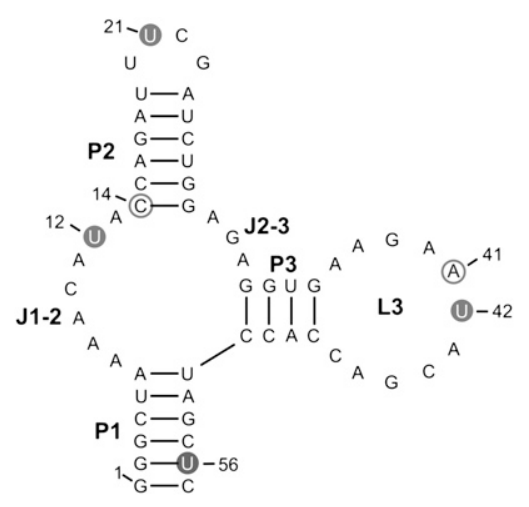

FIGURE 1. Secondary structure predicted for the Tc aptamer according to Hanson et al. (2005). Thiouridines (U) spin labeled with MTS are marked by full gray circles, $2^{\prime}$-aminouridine labeled with 4 -isocyanato-TEMPO are depicted by open gray circles.
(Jeschke and Polyhach 2007) showing its high sensitivity toward small structural changes.

In the present study, we apply this technique to examine the dynamics and conformational transition of the spin labeled Tc aptamer upon ligand binding. Our results reveal the presence of a thermodynamic equilibrium between two aptamer conformations in the free state and a shift toward one conformation upon Tc binding.

\section{RESULTS}

The aim of the present study is to probe the conformational changes of the Tc aptamer upon ligand binding. Therefore, different sites (cf. Fig. 1) were selected for post-synthetic introduction of either the (1-oxyl-2,2,5,5-tetramethylpyrroline-3-methyl) methanethiosulfonate spin label (MTS) by reaction with 4-thiouridine modified RNA (s4U) or of 4-isocyanato-2,6-tetramethylpiperidyl-N-oxid (4-isocyanatoTEMPO) by reaction with 2 '-aminouridine-modified RNA as shown in Figure 2. Due to sterical reasons, the approach involving the $2^{\prime}$-aminouridine modified RNA leads to a smaller disturbance of the bound spin label to ligand binding, which is evident in the binding constant (see Table 1). The conformations and conformational changes of the overall aptamer structure were determined from interspin distances and distance changes within four RNA double mutants, s4U12/s4U21, s4U12/s4U56, s4U42/s4U56, and $C^{\prime} 14 /$ $\mathrm{A}^{\prime} 41$. Spin labeled RNAs are named according to their spin labeled sites. The labeled nucleotide positions are indicated in the secondary structure shown in Figure 1. The double mutants s4U12/s4U21 and s4U12/s4U56 were chosen to study possible conformational changes of the stem regions $\mathrm{P} 1$ and $\mathrm{P} 2$ with respect to the joint $\mathrm{J} 1-2$. The interspin distance of $s 4 \mathrm{U} 42 / \mathrm{s} 4 \mathrm{U} 56$ is sensitive to conformational rearrangement of the opposing loop region with respect to the stem region $\mathrm{P} 1$. Movement of the loop L3 with respect to the single-stranded region J1-2 may be followed with the RNA double-mutant C'14/A'41 with the spin label bound to the RNA sugar-phosphate backbone.

The Tc-binding constants for the double mutants were determined by fluorescence titration spectroscopy to evaluate influences of spin labeling on ligand binding (see Table 1). For $C^{\prime} 14 / A^{\prime} 41$ and s $4 U 42 / s 4 U 56$, the Tc-binding constants resemble those of the unmodified RNA. Doublemutants s4U12/s4U21 and s4U12/s4U56 show a decrease in the binding affinity. This is attributed to the spin label on position 12, as sites 21 and 56 are not part of the binding pocket. U12 does not directly contact Tc, but is involved in the organization of the overall structure of the aptamer by hydrogen bonding to G31 (Xiao et al. 2008). This contact might be disturbed in the spin labeled aptamer conformation. However, it has been shown that nucleotide exchanges on position 12 retain the regulatory activity of the aptamer (Hanson et al. 2005), and the Tc-binding constants for 
A

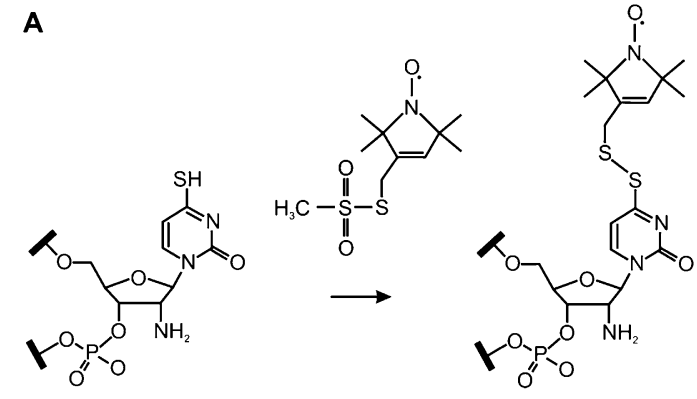

B

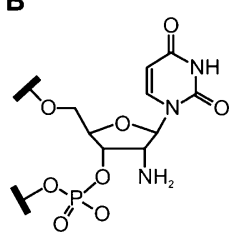

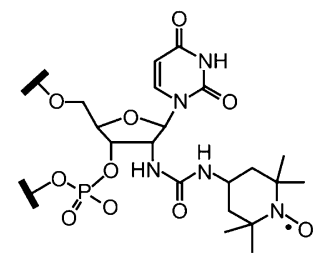

FIGURE 2. Strategy for the post-synthetic introduction of MTS using the selective reaction with 4-thiouridine $(A)$ and of 4-isocyanatoTEMPO using the selective reaction with $2^{\prime}$-aminouridine $(B)$.

double mutants involving s4U12 are still in the nanomolar range (cf. Table 1).

The EPR spectra of the single mutants labeled with MTS (Fig. 3A) are characterized by high motional freedom of the spin label side chains and the presence of only a single motional component. A decreased motional freedom of the TEMPO spin label side chain at $\mathrm{A}^{\prime} 41$ is obvious by the decreased amplitude ratio of the high-field to the middlefield resonance peaks (Fig. 3B). For all single mutants the spin label mobility does not change upon Tc binding, which indicates that the reorientational motion of the spin labels is not influenced by the ligand. Also, for the double mutants (Fig. 3C,D) EPR spectral changes upon ligand binding were not observed.

DEER experiments were performed in the absence and in the presence of Tc, the corresponding results are depicted in Figure 4. In Table 1 the values of the dominating interspin distances and of the estimated distribution widths FWHM are given. For $s 4 \mathrm{U} 12 / \mathrm{s} 4 \mathrm{U} 21$ in the absence of $\mathrm{Tc}$, a distance distribution with two shoulders centered at $2.9 \mathrm{~nm}( \pm 0.5 \mathrm{~nm})$ and $3.8 \mathrm{~nm}( \pm 0.4$ $\mathrm{nm}$ ) is obvious. Binding of Tc reduces the population of the larger distance, and the average distance is shifted to a smaller value. The distance distribution for s4U12/s4U56 in the absence of Tc reveals two major peaks centered at $3.8 \mathrm{~nm}( \pm 0.4 \mathrm{~nm})$ and $5.0 \mathrm{~nm}( \pm 0.5$ $\mathrm{nm})$, whereas in the presence of Tc a well-defined single population with a mean distance of $4.9 \mathrm{~nm}( \pm 0.2 \mathrm{~nm})$ is evident. The experimental result in the absence of $\mathrm{Tc}$ indicates the presence of

a thermodynamic equilibrium either of two conformers of the spin label or of two aptamer conformations. Sites 12 and 56 are highly exposed and the spin label side chains do not experience any restriction of their reorientational motion due to the interaction with the ligand (cf. Figs. 3,5). Room temperature EPR spectra of s4U12, s4U56, and s4U12/s4U56 reveal only a single spectral component, and the spectral shapes are not altered upon ligand binding. Hence, the observed distance distribution is attributed to an equilibrium of two aptamer conformations that characterize the free state of the aptamer. Addition of Tc traps the aptamer conformation corresponding to the larger interspin distance peak. For s4U42/s4U56 the distance distributions are almost identical in the presence and absence of Tc, with a mean distance of $4.8 \mathrm{~nm}( \pm 0.2 \mathrm{~nm})$. A manual variation of the homogenous background correction shifts the mean distances by $<0.4 \mathrm{~nm}$ for these mutants. The data for $\mathrm{C}^{\prime} 14 / \mathrm{A}^{\prime} 41$ in the absence of Tc exhibits three major peaks centered at $2.6 \mathrm{~nm}( \pm 0.3 \mathrm{~nm}), 3.4 \mathrm{~nm}( \pm 0.2 \mathrm{~nm})$, and $4.7 \mathrm{~nm}( \pm 0.7$ $\mathrm{nm})$. In the presence of $\mathrm{Tc}$ the population ratio of the peaks corresponding to $2.6 \mathrm{~nm}$ and $3.4 \mathrm{~nm}$ are interconverted. Variation of the homogenous background correction leads to a significant decrease in the population for distances larger than $4.5 \mathrm{~nm}$. This is strong evidence that the peaks at $4.8 \mathrm{~nm}$ do not reflect dipolar interaction within the same RNA molecule, but are due to the interaction with background spins or have to be attributed to the presence of a fraction of unfolded aptamers. In addition to the analysis by Tikhonov regularization, all data were fitted with a Monte Carlo/SIMPLEX algorithm assuming a sum of Gaussian-distributed conformers contributing to the dipolar evolution data by use of the software DEFit 3.9 (Sen et al. 2007). The resulting distance distributions (data not shown) resemble those obtained by Tikhonov regularization.

The location and the microenvironment of the introduced spin labels are schematically represented in the aptamer model depicted in Figure 5. Regions J1-2, J2-3, and L3 of the model were built according to the X-ray structure of the circularly permuted tetracycline-dependent aptamer (Xiao et al. 2008). The model was complemented
TABLE 1. Binding constants $K_{D}$ of double labeled RNA mutants and interspin distances determined in the absence and in the presence of tetracycline (Tc)

\begin{tabular}{lccccc}
\hline & wild type & s4U12/s4U21 & s4U12/s4U56 & s4U42/s4U56 & $\mathrm{C}^{\prime} 14 / \mathrm{A}^{\prime} 41$ \\
\hline $\mathrm{K}_{\mathrm{D}} / \mathrm{nM}$ & $0.77 \pm 0.11^{\mathrm{a}}$ & $137 \pm 10$ & $103 \pm 16$ & $6.2 \pm 0.7$ & $4.2 \pm 0.2$ \\
Interspin distance & $2.9 \pm 0.5$ & $3.8 \pm 0.4$ & $4.8 \pm 0.2$ & $2.6 \pm 0.3$ \\
without Tc/nm & $3.8 \pm 0.4$ & $5.0 \pm 0.5$ & & $(3.4 \pm 0.2)$ \\
Interspin distance & $(2.3 \pm 0.4)$ & $4.9 \pm 0.2$ & $4.7 \pm 0.2$ & $(2.7 \pm 0.4)$ \\
with Tc/nm & $2.8 \pm 0.5$ & & & $3.6 \pm 0.3$ \\
& $(3.8 \pm 0.5)$ & & & \\
\hline
\end{tabular}

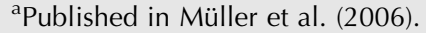

Dominating interspin distance peaks are given with their estimated FWHM widths, peaks with decreased probability, or shoulders are shown in parentheses. 

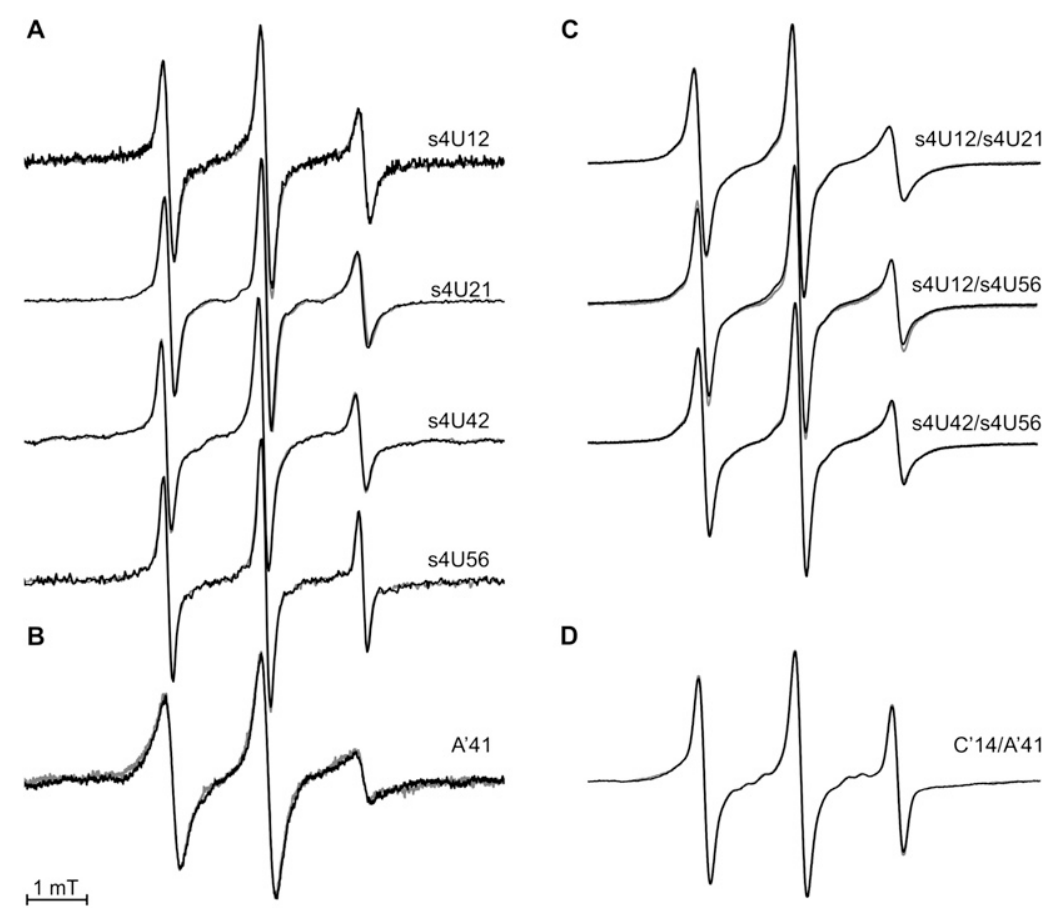

FIGURE 3. Room temperature continuous wave EPR spectra measured at X-band in the absence (black lines) and in the presence of Tc (gray lines). ( $A, C)$ Single and double mutants labeled with MTS and $(B, D)$ single and double mutants labeled with the 4-isocyanato-TEMPO. All plots are normalized by amplitude.

with a schematic representation of the stems P1 and P2 based on the chemical and enzymatic probing experiments (Hanson et al. 2005) according to the experimental interspin distances. The experimentally determined interspin distances are indicated by gray-dotted arrows in Figure 5A. The two components in the distance distributions of s4U12/s4U21, s4U12/s4U56, and $\mathrm{C}^{\prime} 14 / \mathrm{A}^{\prime} 41$ represent two locations of the nitroxides at positions 12 and 14 as indicated. The displacement of these nitroxides could not be achieved by reorientation of the nitroxide side chains, but required adjusting of region J1-2 as represented in Figure 5B. Upon ligand binding, one conformation (blue) is trapped that resembles that of the $\mathrm{X}$-ray structure (3EGZ).

\section{DISCUSSION}

The aim of this study was to investigate the dynamics and possible conformational changes of the Tc aptamer upon ligand binding. Therefore, different single and double spin labeled aptamer mutants were synthesized and analyzed by EPR spectroscopy. The results for the double mutants s4U12/s4U21, s4U12/s4U56, and $\mathrm{C}^{\prime} 14 / \mathrm{A}^{\prime} 41$ indicate the presence of a thermodynamic equilibrium of two aptamer conformations characteristic of the free state of the aptamer. Ligand-binding shifts this equilibrium toward one of these conformations. The spin label at site 21 is highly exposed without any restriction in its reorientational motion or ligand interaction according to the model illustrated in Figure 5. In addition, the single component room-temperature EPR spectra do not reveal any evidence for interaction with the ligand. Site 56 is located in stem $\mathrm{P} 1$, which is supposed to form the scaffold of the aptamer without direct ligand interaction. Since site 12 is located in the single-stranded region J1-2, the distance changes observed for s4U12/s4U21 and s4U12/ s4U56 indicate a displacement of this region upon Tc binding as displayed in Figure 5 . The results of $s 4 \mathrm{U} 42 / \mathrm{s} 4 \mathrm{U} 56$ reveal that $s 4 \mathrm{U} 42$ is not involved in conformational changes upon ligand binding; the distance distributions in the presence and absence of Tc are identical. Hence, a ligand-induced transformation of the opposing loop region along the stem region P1 is unlikely, and a transformation of the stem regions of the aptamer could not be resolved. Therefore, the distance changes detected for $C^{\prime} 14 / A^{\prime} 41$ again reveal the displacement of the single-stranded region J1-2. In conclusion, addition of Tc leads to the capture of one aptamer conformation that resembles that of the X-ray structure (Xiao et al. 2008).

Similar conformational changes have been revealed by NMR spectroscopy for a citrulline-binding RNA aptamer and the related arginine-binding aptamer (Yang et al. 1996). Both aptamers contain two asymmetrical internal loops that are not well ordered in the free RNA, but fold into a compact structure upon ligand binding, with one nucleotide capping the binding pocket. Evidence for a dynamic equilibrium of an "inactive" and an "active" state was provided for a theophylline-binding RNA aptamer (Latham et al. 2009). Their results indicated that the RNA utilize a conformational selection mechanism for binding theophylline like other RNAs with conformationally heterogeneous free states, such as the aptamer domains in RNA riboswitches binding their ligands. Furthermore, a previous NMR study on the aptamer domain of a guanine riboswitch (Noeske et al. 2006) revealed that the aptamer domain binds the ligand in a disordered and unstructured binding pocket, but with a preorganized tertiary interaction between two loops in its free state. The loop-loop interaction organizes the global fold of the RNA prior to ligand binding, only small changes are necessary to accommodate the ligand.

In this work we studied the conformational transition of a spin labeled Tc-binding RNA aptamer using DEER spectroscopy in combination with site-directed spin labeling. We have focused on the determination of interspin distances of four double spin labeled aptamer variants, 


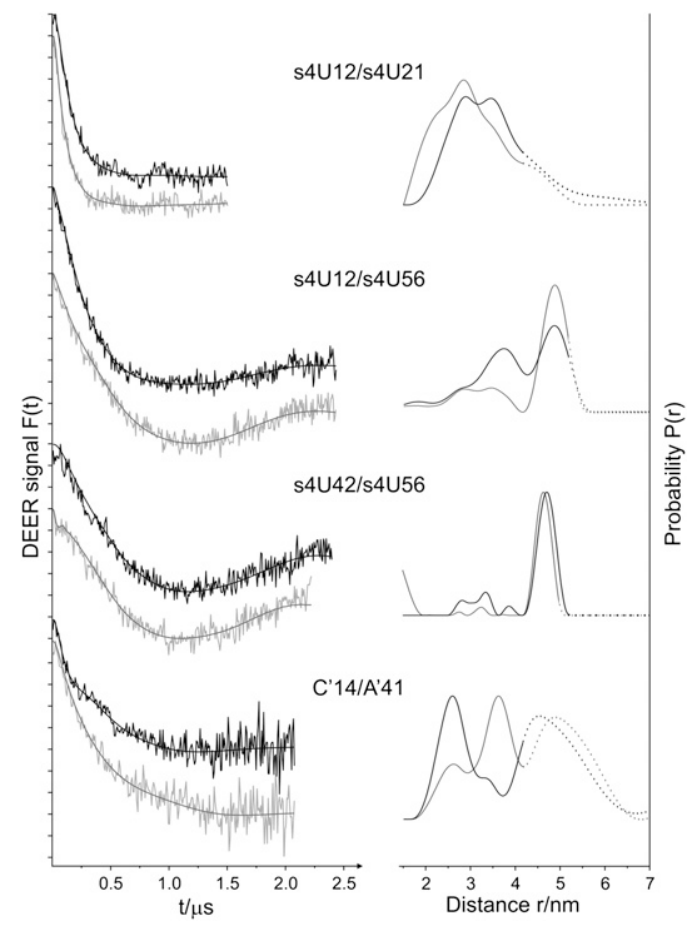

FIGURE 4. DEER analysis of the RNA double mutants s4U12/s4U21, s4U12/s4U56, s4U42/s4U56, and $\mathrm{C}^{\prime} 14 / \mathrm{A}^{\prime} 41$ in the absence (black) and in the presence of Tc (gray). (Left) Background-corrected dipolar evolution data $\mathrm{F}(t)$. Tick marks are separated by 0.02 . (Right) Distance distributions $\mathrm{P}(r)$ obtained using DeerAnalysis2006 (Jeschke et al. 2006). In the dotted range, intermolecular spin-spin interaction may contribute.

which uncovers a thermodynamic equilibrium of two aptamer conformations in the absence of $\mathrm{Tc}$ and the capture of one conformation in presence of Tc. Existence of a conformational equilibrium in the free state and shift to one conformation upon ligand binding seems to be a capable mechanism for a riboswitch to provide the necessary action to impact on gene expression.

\section{MATERIALS AND METHODS}

\section{Synthesis and spin labeling of RNA aptamers}

The RNA variants were obtained by solid-phase RNA synthesis using standard phosphoramidite chemistry as described (Schmidt et al. 2000; Welz and Müller 2002). 3'-O-phosphoramidites of $\mathrm{N}$-acyl-5'-O-dimethoxytrityl-2'-O-tertbutyldimethylsilyl protected nucleosides were obtained from ChemGenes. A set of different positions in the stems, single-stranded region and loop region was selected for post-synthetically spin labeling (Fig. 1; highlighted in gray) with either the (1-oxyl-2,2,5,5-tetramethylpyrroline3-methyl)methanethiosulfonate (MTS) spin label or the 4-isocyanato-2,6-tetramethylpiperidyl-N-oxid (4-isocyanato-TEMPO) spin label (Fig. 2).

The modified nucleotide 4-thiouridine was incorporated at preselected positions, instead of uridine, to be used for postsynthetic spin labeling with MTS (Qin et al. 2003). RNA samples were incubated for $2 \mathrm{~h}$ at room temperature (RT) with a 200 -fold excess of dithiothreitol (DTT) in $50 \mu \mathrm{L}$ of $100 \mathrm{mM}$ sodium phosphate buffer $(\mathrm{pH} 8.0)$. The reducing agent was removed by demineralization using centrifugal filters, followed by lyophilization of the RNA. Spin labeling was carried out overnight by incubation of a $100 \mu \mathrm{M}$ solution of the RNA double mutant in $90 \mathrm{mM}$ sodium phosphate buffer $(90 \% \mathrm{v} / \mathrm{v})(\mathrm{pH} 8.0)$ and dimethylformamide (DMF) (10\% v/v) with a 10 -fold excess of MTS. Unbound MTS was removed by demineralization and lyophilization as described above. Different singly and doubly spin labeled RNAs (named according to the spin labeled sites) were synthesized, namely, single mutants s4U12, s4U21, s4U 42 , and s4U56 and double mutants s4U12/s4U21, s4U12/s4U56, and s4U42/ s4U56.

Alternatively, 4-isocyanato-TEMPO was attached to the sugar moiety of the specific nucleotides. Therefore, RNA mutants were synthesized with 2 '-aminouridine and subsequently spin labeled (Edwards et al. 2001; Kim et al. 2004). A $100 \mu \mathrm{M}$ solution of the RNA in $100 \mathrm{mM}$ sodium borate buffer ( $\mathrm{pH} 8.5)(50 \% \mathrm{v} / \mathrm{v})$, DMF $(20 \% \mathrm{v} / \mathrm{v})$ and formamide $(30 \% \mathrm{v} / \mathrm{v})$ was cooled down to $-8^{\circ} \mathrm{C}$ and incubated for $2 \mathrm{~h}$ with a 15 -fold excess of TEMPO. Side products and unbound reagents were extracted with $300 \mu \mathrm{L}$ of chloroform at RT, followed by precipitation of the labeled RNA from ethanol, resolving the pellet in water, demineralization using centrifugal filters, and finally, lyophilization of the RNA. Again, labeled RNAs are named according to the spin labeled sites,

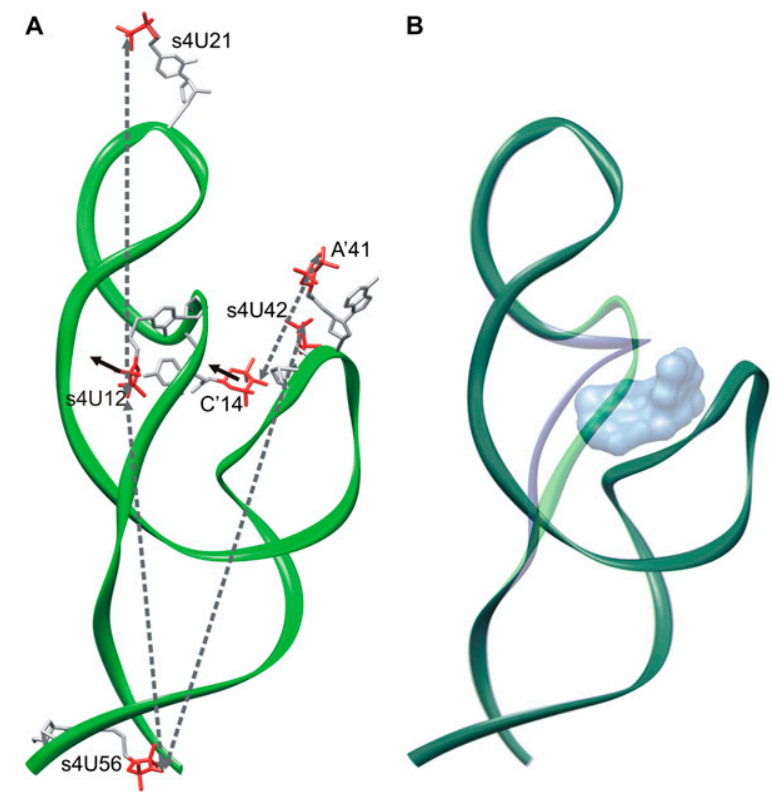

FIGURE 5. Schematic representations of the Tc aptamer conformations. The regions $\mathrm{J} 1-2, \mathrm{~J} 2-3$, and L3 of the X-ray structure of the circularly permuted Tc-dependent aptamer (Xiao et al. 2008) was complemented with a schematic representation of the stems P1 and P2 based on the chemical and enzymatic probing experiments (Hanson et al. 2005). (A) Interspin distances between nitroxides at positions $12,14,21,41,42$, and 56 , as indicated by gray-dotted arrows were used as constraints during modeling. Black arrows indicate the displacement of the nitroxides at positions 12 and 14 upon addition of Tc. (B) Two aptamer conformations (green, blue) are in equilibrium in the free state. Upon addition of Tc (shown in surface representation) the aptamer conformation depicted in blue is trapped. 
namely, $C^{\prime} 14$ for the single mutant and $C^{\prime} 14 / A^{\prime} 41$ for the double mutant. For both spin label strategies the spin label efficiency was approximately above $50 \%$.

\section{Fluorescence titration experiments}

Fluorescence titration experiments were carried out at $25^{\circ} \mathrm{C}$ on a Fluorolog FL3-22. The excitation wavelength was set to $370 \mathrm{~nm}$. Spectra were acquired from 450 to $600 \mathrm{~nm}$ in $1 \mathrm{~nm}$ increments with an integration time of $0.3 \mathrm{sec}$. Slits were set to $2 \mathrm{~mm}$. Tc was presented in a total volume of $2 \mathrm{~mL}$ of binding buffer $(20 \mathrm{mM}$ potassium phosphate buffer at $\mathrm{pH} 7.5,100 \mathrm{mM} \mathrm{NaCl}, 1 \mathrm{mM}$ $\mathrm{MgCl}_{2}$ ). The RNA was dissolved in binding buffer and titrated, avoiding a total volume increase of $>5 \%$. The solution was stirred during each titration step and allowed to equilibrate for $2 \mathrm{~min}$ before data collection. Fluorescence spectra were measured for each titration point. The peak area of the fluorescence emission signal was averaged, normalized, and plotted against the free RNA concentration. The dissociation constant $\mathrm{K}_{\mathrm{D}}$ was calculated as described earlier (Müller et al. 2006).

\section{EPR sample preparation}

The RNA was dissolved (50-70 $\mu \mathrm{M}$ final concentration for single mutants or $110-150 \mu \mathrm{M}$ for double mutants) in $20 \mathrm{mM}$ potassium phosphate buffer, $100 \mathrm{mM} \mathrm{NaCl}, 5 \mathrm{mM} \mathrm{MgCl}_{2}(\mathrm{pH}$ 7.5). A total of $5 \%-20 \%$ glycerol $(\mathrm{v} / \mathrm{v})$ was added as a cryoprotectant and deuterated water was used to increase the transverse relaxation time for pulse EPR experiments. The RNA mutants were incubated at $45^{\circ} \mathrm{C}$ for $5 \mathrm{~min}$, followed by slow cooling to RT in order to allow homogeneous tertiary structure formation. Complex formation with a twofold excess of Tc was assured by an additional incubation for $5 \mathrm{~min}$ at $37^{\circ} \mathrm{C}$ and slow cooling to RT.

For the DEER experiments $30-40 \mu \mathrm{L}$ of the sample solution was filled into EPR quartz capillaries and frozen in liquid nitrogen before insertion into the resonator. Sample volumes of $10 \mu \mathrm{L}$ were loaded into EPR quartz capillaries for continuous wave (cw) EPR measurements.

\section{EPR setup}

Room temperature $\mathrm{cw}$ EPR spectra at X-band were recorded using a Magnettech Miniscope MS200 X-band spectrometer equipped with a rectangular TE102 resonator. To avoid saturation and to obtain high signal-to-noise ratio, the microwave power was set to $10 \mathrm{~mW}$ and the B-field modulation amplitude was adjusted to $0.15 \mathrm{mT}$.

All DEER experiments were performed at $50 \mathrm{~K}$ using a Bruker Elexsys 580 spectrometer equipped with a 3-mm split ring resonator (ER 4118X-MS3; Bruker) at X-band frequencies (9.4 $\mathrm{GHz}$ ). The resonator was overcoupled to $\mathrm{Q} \sim 100$. Temperature stabilization was achieved by a continuous-flow helium cryostat (ESR 900; Oxford Instruments) in combination with a temperature controller (ITC 503S; Oxford Instruments). The following four-pulse DEER sequence was used:

$$
\begin{aligned}
& \pi / 2\left(v_{o b s}\right)-\tau_{1}-\pi\left(v_{o b s}\right)-t^{\prime}-\pi\left(v_{p u m p}\right)-\left(\tau_{1}+\tau_{2}-t^{\prime}\right) \\
& \quad-\pi\left(v_{o b s}\right)-\tau_{2}-e c h o
\end{aligned}
$$

(Pannier et al. 2000)

For all pulses at the observer frequency the $\langle\mathrm{X}\rangle$ channels were applied. A two-step phase cycling $(+\langle\mathrm{X}\rangle,-\langle\mathrm{X}\rangle)$ was performed on $\pi / 2\left(v_{\text {obs }}\right)$. The observer pulses had lengths of $16 \mathrm{nsec}$ for $\pi / 2$ and $32 \mathrm{nsec}$ for $\pi$ pulses, and the pump pulse length was set to $12 \mathrm{nsec}$. The pump frequency, $v_{\text {pump}}$, was positioned at the center of the resonator dip. This frequency corresponded to the maximum of the echo-detected nitroxide EPR absorption spectrum. The observer frequency, $v_{\text {obs }}$, was set to the low-field local maximum of the absorption spectrum, which resulted in a $65 \mathrm{MHz}$ frequency offset. Time $\tau_{1}$ and $\tau_{2}$ were kept constant, whereas $t^{\prime}$ was varied. Deuterium modulation was averaged by addition of traces at eight different $\tau_{1}$ start values, starting at $\tau_{1,0}=400 \mathrm{nsec}$ and incrementing by $\Delta \tau_{1}=56 \mathrm{nsec}$. The dipolar evolution time was given by $t=$ $t^{\prime}-\tau_{1}$ and data with $t>0$ were analyzed. The experimental echo decay was background corrected using a homogeneous threedimensional spin distribution. The experimental DEER spectra yield information about intra- and intermolecular interspin distances. A background correction separates the intermolecular background contribution from the intramolecular contribution. Interspin distance distributions were derived by fitting the dipolar evolution function using Tikhonov regularization, a commonly used method of regularization of ill-posed problems, as implemented in DEERAnalysis2006 (Jeschke et al. 2006). In addition, the experimental data were fitted with a Monte Carlo/SIMPLEX algorithm assuming a sum of Gaussian-distributed interspin distances contributing to the dipolar evolution data by use of the software DEFit 3.9 (Sen et al. 2007).

\section{Molecular modeling}

The starting structure for molecular modeling was the X-ray structure of the circularly permuted tetracycline aptamer (Protein Data Bank code: 3EGZ) (Xiao et al. 2008). The regions J1-2, J2-3, and loop L3 were extracted and complemented with the stems $\mathrm{P} 1$ and P2. Both stems were constructed according to the chemical and enzymatic probing experiments (Hanson et al. 2005). Spin labels were introduced at the positions $12,14,21,41,42$, and 56; the topologies for the spin label side chains were set up with respect to published data (Beier and Steinhoff 2006) and similar configurations in the Gromacs library. The initial molecular model was adapted on the bases of the experimentally determined interspin distances, resulting in two molecular models that correspond to the two aptamer conformations in the free state. For both molecular models, energies were minimized by means of steepest descent using the amber99 force field (Sorin and Pande 2005) implemented in the Gromacs simulation package (Van der Spoel et al. 2005). Periodic boundary conditions in the presence of TIP3P water molecules were utilized.

\section{ACKNOWLEDGMENTS}

We gratefully acknowledge funding by the Volkswagenstiftung (I/ 79 950-952).

Received April 15, 2010; accepted October 27, 2010.

\section{REFERENCES}

Beier C, Steinhoff HJ. 2006. A structure-based simulation approach for electron paramagnetic resonance spectra using molecular and stochastic dynamics simulations. Biophys J 91: 2647-2664.

Berens C, Hillen W. 2003. Gene regulation by tetracyclines. Eur J Biochem 270: 3109-3121. 
Bordignon E, Steinhoff HJ. 2007. Membrane protein structure and dynamics studied by site-directed spin labeling ESR. In ESR spectroscopy in membrane biophysics (ed. Hemminga MA, Berliner LJ), pp. 129-164. Springer Science and Business Media, New York.

Cai Q, Kusnetzow AK, Hubbell WL, Haworth IS, Gacho GPC, Van Eps N, Hideg K, Chambers EJ, Qin PZ. 2006. Site-directed spin labeling measurements of nanometer distances in nucleic acids using a sequence-independent nitroxide probe. Nucleic Acids Res 34: 4722-4730.

Edwards TE, Okonogi TM, Robinson BH, Sigurdsson ST. 2001. Sitespecific incorporation of nitroxide spin-labels into internal sites of the TAR RNA; structure-dependent dynamics of RNA by EPR spectroscopy. J Am Chem Soc 123: 1527-1528.

Ellington AD, Szostak JW. 1990. In vitro selection of RNA molecules that bind specific ligands. Nature 346: 818-822.

Epe B, Woolley P, Hornig H. 1987. Competition between tetracycline and tRNA at both P and A site of the ribosome of Escherichia coli. FEBS Lett 213: 443-447.

Famulok M. 1999. Oligonucleotide aptamers that recognize small molecules. Curr Opin Struct Biol 9: 324-329.

Famulok M, Hartig JS, Mayer G. 2007. Functional aptamers and aptazymes in biotechnology, diagnostics, and therapy. Chem Rev 107: 3715-3743.

Gold L, Polisky B, Uhlenbeck O, Yarus M. 1995. Diversity of oligonucleotide functions. Annu Rev Biochem 64: 763-797.

Hanson S, Bauer G, Fink B, Suess B. 2005. Molecular analysis of a tetracycline-binding riboswitch. RNA 11: 503-511.

Hermann T, Patel DJ. 2000. Adaptive recognition by nucleic acid aptamers. Science 287: 820-825.

Hubbell WL, Cafiso DS, Altenbach C. 2000. Identifying conformational changes with site-directed spin labeling. Nat Struct Biol 7: 735-739.

Jeschke G, Polyhach Y. 2007. Distance measurements on spin-labelled biomacromolecules by pulsed electron paramagnetic resonance. Phys Chem Chem Phys 9: 1895-1910.

Jeschke G, Chechik V, Ionita P, Godt A, Zimmermann H, Banham J, Timmel CR, Hilger D, Jung H. 2006. DeerAnalysis2006 - a comprehensive software package for analyzing pulsed ELDOR data. Appl Magn Reson 30: 473-498.

Kim HD, Nienhaus GU, Ha T, Orr JW, Williamson JR, Chu S. 2002. $\mathrm{Mg}^{2+}$-dependent conformational change of RNA studied by fluorescence correlation and FRET on immobilized single molecules. Proc Natl Acad Sci 99: 4284-4289.

Kim NK, Murali A, DeRose VJ. 2004. A distance ruler for RNA using EPR and site-directed spin labeling. Chem Biol 11: 939-948.

Kötter P, Weigand JE, Meyer B, Entian KD, Suess B. 2009. A fast and efficient translational control system for conditional expression of yeast genes. Nucleic Acids Res 18: e120. doi: 10.1093/nar/gkp578.

Latham MP, Zimmermann GR, Pardi A. 2009. NMR chemical exchange as a probe for ligand-binding kinetics in a theophylline-binding RNA aptamer. J Am Chem Soc 131: 5052-5053.

Lorenz M, Diekmann S. 2006. Distance determination in proteinDNA complexes using fluorescence resonance energy transfer. Methods Mol Biol 335: 243-255.

Margittai M, Widengren J, Schweinberger E, Schroder GF, Felekyan S, Haustein E, Konig M, Fasshauer D, Grubmuller H, Jahn R, et al. 2003. Single-molecule fluorescence resonance energy transfer reveals a dynamic equilibrium between closed and open conformations of syntaxin 1. Proc Natl Acad Sci 100: 1551615521.

Müller M, Weigand JE, Weichenrieder O, Suess B. 2006. Thermodynamic characterization of an engineered tetracycline-binding riboswitch. Nucleic Acids Res 34: 2607-2617.

Noeske J, Buck J, Fürtig B, Nasiri HR, Schwalbe H, Wöhnert J. 2006. Interplay of 'induced fit' and preorganization in the ligand induced folding of the aptamer domain of the guanine binding riboswitch. Nucleic Acids Res 35: 572-583.
Pannier M, Veit S, Godt A, Jeschke G, Spiess HW. 2000. Dead-time free measurement of dipole-dipole interactions between electron spins. J Magn Reson 142: 331-340.

Patel DJ, Suri AK. 2000. Structure, recognition and discrimination in RNA aptamer complexes with cofactors, amino acids, drugs and aminoglycoside antibiotics. J Biotechnol 74: 39-60.

Piton N, Schiemann O, Mu Y, Stock G, Prisner T, Engels JW. 2005. Synthesis of spin-labeled RNAs for long range distance measurements by PELDOR. Nucleosides Nucleotides Nucleic Acids 24: 771-775.

Qin PZ, Hideg K, Feigon J, Hubbell WL. 2003. Monitoring RNA base structure and dynamics using site-directed spin labeling. Biochemistry 42: 6772-6783.

Roth A, Breaker RR. 2009. The structural and functional diversity of metabolite-binding riboswitches. Annu Rev Biochem 78: 305-334.

Schiemann O, Weber A, Edwards TE, Prisner T, Sigurdsson ST. 2003. Nanometer distance measurements on RNA using PELDOR. J Am Chem Soc 125: 3434-3435.

Schiemann O, Piton N, Mu Y, Stock G, Engels JW, Prisner T. 2004. A PELDOR-based nanometer distance ruler for oligonucleotides. J Am Chem Soc 126: 5722-5729.

Schmidt C, Welz R, Müller S. 2000. RNA double cleavage by a hairpin-derived twin ribozyme. Nucleic Acids Res 28: 886-894.

Sen KI, Logan TM, Fajer PG. 2007. Protein dynamics and monomermonomer interactions in AntR activation by electron paramagnetic resonance and double electron-electron resonance. Biochemistry 46: 11639-11649.

Sicoli G, Mathis G, Delalande O, Boulard Y, Gasparutto D, Gambarelli S. 2008. Double electron-electron resonance (DEER): A convenient method to probe DNA conformational changes. Angew Chem Int Ed Engl 47: 735-737.

Sicoli G, Mathis G, Aci-Sèche S, Saint-Pierre C, Boulard Y, Gasparutto D, Gambarelli S. 2009. Lesion-induced DNA weak structural changes detected by pulsed EPR spectroscopy combined with site-directed spin labeling. Nucleic Acids Res 37: 3165-3176.

Sorin EJ, Pande VS. 2005. Exploring the helix-coil transition via allatom equilibrium ensemble simulations. Biophys J 88: 2472-2493.

Spahn CMT, Prescott CD. 1996. Throwing a spanner in the works: Antibiotics and the translation apparatus. J Mol Med 74: 423-439.

Suess B, Weigand JE. 2008. Engineered riboswitches: Overview, problems and trends. RNA Biol 5: 24-29.

Suess B, Hanson S, Berens C, Fink B, Schroeder R, Hillen W. 2003. Conditional gene expression by controlling translation with tetracycline-binding aptamers. Nucleic Acids Res 31: 1853-1858.

Tuerk C, Gold L. 1990. Systematic evolution of ligands by exponential enrichment: RNA ligands to Bacteriophage T4 DNA polymerase. Science 249: 505-510.

Van der Spoel D, Lindahl E, Hess B, Groenhof G, Mark AE, Berendsen HJC. 2005. GROMACS: Fast, flexible, and free. J Comput Chem 26: $1701-1718$.

Weigand JE, Suess B. 2007. Tetracycline aptamer-controlled regulation of pre-mRNA splicing in yeast. Nucleic Acid Res 35: 4179-4185.

Weigand JE, Suess B. 2009. Aptamers and riboswitches: Perspective in biotechnology. Appl Microbiol Biotechnol 85: 229-236.

Welz R, Müller S. 2002. 5-(Benzylmercapto)-1H-tetrazole as activator for 2'-O-TBDMS phosphoramidite building blocks in RNA synthesis. Tetrahedron Lett 43: 795-797.

Wilson DS, Szostak JW. 1999. In vitro selection of functional nucleic acids. Annu Rev Biochem 68: 611-647.

Win MN, Liang JC, Smolke CD. 2009. Frameworks for programming biological function through RNA parts and devices. Chem Biol 16: 298-310.

Xiao H, Edwards TE, Ferré-D'Amaré AR. 2008. Structural basis for specific, high-affinity tetracycline binding by an in vitro evolved aptamer and artificial riboswitch. Chem Biol 15: 1125-1137.

Yang Y, Kochoyan M, Burgstaller P, Westhof E, Famulok M. 1996. Structural basis of ligand discrimination by two related RNA aptamers resolved by NMR spectroscopy. Science 272: 1343-1347. 

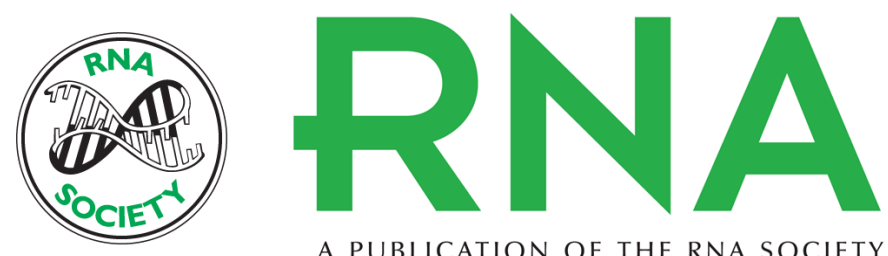

A PUBLICATION OF THE RNA SOCIETY

\section{Ligand-induced conformational capture of a synthetic tetracycline riboswitch revealed by pulse EPR}

Dorith Wunnicke, Denise Strohbach, Julia E. Weigand, et al.

RNA 2011 17: 182-188 originally published online November 19, 2010

Access the most recent version at doi:10.1261/rna.2222811

$\begin{array}{ll}\text { References } & \begin{array}{l}\text { This article cites } 46 \text { articles, } 6 \text { of which can be accessed free at: } \\ \text { http://rnajournal.cshlp.org/content/17/1/182.full.html\#ref-list-1 }\end{array}\end{array}$

License

Email Alerting Receive free email alerts when new articles cite this article - sign up in the box at the Service top right corner of the article or click here.

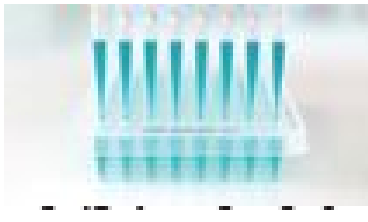

Providing Precise Solutions for your research.

To subscribe to RNA go to:

http://rnajournal.cshlp.org/subscriptions 